\title{
Attitude Maneuver Tests Using CMGs Mounted in a Three-Axis Free Dynamics Simulator
}

\author{
By Takuya KanZawA, ${ }^{1)}$ Misuzu HaruKI,${ }^{1)}$ Tatsuya Endo ${ }^{1), 2)}$ and Koji YAmAnAKA ${ }^{1)}$ \\ ${ }^{1)}$ Japan Aerospace Exploration Agency, Tsukuba, Japan \\ ${ }^{2)}$ Currently, Mitsubishi Electric Corporation, Kamakura, Japan
}

(Received July 28th, 2015)

\begin{abstract}
This paper presents attitude maneuver demonstration tests using control moment gyroscopes mounted in a three-axis free dynamics simulator that provides an on-ground test environment of free rotational movement around three axes using the air-floating method. The issue of torque error between the torque command and the actual output torque is discussed in relation to the singularity-robust based steering law for spacecraft maneuvers. The torque error is analyzed using singular value decomposition to determine its magnitude and direction. Based on the analysis, feedback control and steering laws are proposed to reduce the torque error. Before conducting the maneuver demonstration test, a preliminary simulation is performed using a simplified dynamics model, control law, and steering law. The maneuver test results indicate that the dynamics simulator successfully accomplishes agile, large angle, and rest-to-rest multitarget maneuvers as well as precision pointing.
\end{abstract}

Key Words: Control Moment Gyroscopes, Dynamics Simulator, Maneuver, Agile, Torque Error

\section{Nomenclature}

\section{A : Jacobian matrix}

$\mathrm{d}_{\mathrm{i}} \quad$ : singularity robustness parameter

H : total angular momentum

$\mathrm{h}_{\mathrm{R}} \quad$ : angular momentum of rotor

I : inertia matrix of the sphere

$\mathrm{K}_{\mathrm{N}} \quad$ : null motion gain

$\mathrm{m}$ : CMG gain

$\mathbf{O}_{\mathrm{nn}} \quad: \mathrm{n} \times \mathrm{n}$ null matrix

$\mathbf{S}_{\mathrm{V}} \quad$ : projection matrix

$\mathbf{T}_{\mathrm{CNT}}$ : torque command

$\mathbf{U}_{\mathrm{nn}}: \mathrm{n} \times \mathrm{n}$ identity matrix

$\mathbf{u}_{\mathrm{d}}$ : actual output torque

$\mathbf{u}_{\text {err }}:$ torque error

$\mathbf{u}_{\mathrm{si}} \quad:$ left singular vector

$\mathbf{v}_{\text {si }}:$ right singular vector

$\mathbf{V} \quad$ : weighting matrix

$\beta \quad$ : CMG skew angle

$\boldsymbol{\delta} \quad$ : gimbal angle

$\sigma_{\mathrm{i}}$ : singular value

$\Theta$ : attitude angle of the sphere

$\omega$ : attitude rate of the sphere

$\xi \quad:$ magnitude of torque error

$\zeta_{\mathrm{i}} \quad:$ magnitude of torque command

Superscript

$\mathrm{T} \quad$ : transpose of matrices

\section{Introduction}

Control moment gyroscopes (CMGs) have recently been deployed on Earth- and astronomy-observing spacecrafts to observe multiple targets using agile attitude maneuvers. Although CMGs can produce large torques in comparison with reaction wheels, difficulties still exist because of $\mathrm{CMG}$ hardware and software complexities. One such complexity is the singularity problem, in which no control torque is produced along a singular direction. Many CMG steering algorithms for avoiding or traversing these singularities have been extensively studied. ${ }^{1,2)}$ In addition to steering algorithms, a test facility is required to demonstrate agile maneuvers for on-orbit use. Validation and verification using such a test facility will ensure that we can develop an attitude control system employing CMGs. Thus, to conduct an on-ground agile maneuver test, we first developed a large dynamics simulator that provides a test environment for free rotational movement around three axes using the air-floating method. ${ }^{3,4)}$

As a steering law for agile, large angle, and rest-to-rest multitarget maneuvers, we propose the combination of the singularity-robust based steering law with null motion. ${ }^{5)}$ However, there is a drawback that the actual output torque produced by the CMG system is not identical to the torque command required for three-axis attitude control in the vicinity of a singularity. Such a torque error between the actual output torque and the torque command causes undesirable attitude errors. If the control law generates a large feedback torque command to compensate for these attitude errors, the steering law will inevitably generate a command with an undesirable excessive gimbal rate. This excessive gimbal rate, involving abrupt changes, will result in gimbal motor load and will unnecessarily consume the gimbal motor's power. Therefore, torque error should be minimized to prevent the gimbal rate from becoming excessive. Jin et al. used the singular value decomposition (SVD) theory to determine the torque error mechanism induced by the singularity-robust based steering law without null motion. ${ }^{6}$ 
This study aims to present control and steering laws designed to mitigate the torque error and demonstrate agile maneuver tests using the simplified control and steering laws, as implemented in the dynamics simulator. The remainder of this paper is as follows: First, in Section 2, the dynamics simulator configuration, as presented by Endo et al. ${ }^{3)}$ and Kanzawa et al. ${ }^{4}$, will be introduced. Second, Section 3 provides an overview of the dynamics model, control law, and steering law proposed by Kanzawa et al. ${ }^{5)}$ Third, in Section 4, the torque error induced by the singularity-robust based steering law with null motion will be analyzed using SVD theory to determine its magnitude and direction. Based on the analysis, feedback control and steering laws will be proposed to produce less torque errors in the vicinity of a singularity. In Section 5, before conducting the maneuver demonstration tests using the dynamics simulator, a preliminary simulation is performed on the basis of the simplified model to predict the behavior of the dynamics simulator and CMGs. Finally, in Section 6, the maneuver demonstration test results are shown, and the tested performance is compared with the predicted performance obtained through numerical simulation.

\section{Dynamics Simulator Configuration}

\subsection{Design concept}

Figure 1 shows the dynamics simulator, which takes into consideration three-axis free rotational motion using the air-floating method. Descriptions for the dynamics simulator and CMGs can be found in Refs. 3) and 4), respectively. The dynamics simulator consists of a large aluminum saucer and sphere, which is approximately $2 \mathrm{~m}$ in diameter and $1400 \mathrm{~kg}$ in weight. The sphere floats in the saucer on compressed air that originates from many small holes embedded in the saucer. Since equipment such as attitude control sensors and actuators can be mounted inside the sphere, unlimited and full $360^{\circ}$ rotational movement can be realized about the three axes; most other test facilities that utilize spherical air bearings are constrained to rotate about one or two axes since their equipment is mounted on the main structure supported by the air bearings. ${ }^{7-9)}$ The sphere can be divided into two sections, i.e., the upper and lower hemispheres. The large volume inside the lower hemisphere accommodates four large single gimbal type CMGs, which are mounted in a pyramid configuration with a skew angle of $\beta=60^{\circ}$ (Fig. 2).

The major disturbance torque exerted on the sphere is the gravitational torque due to the offset between the center of mass and rotation. This offset causes an undesirable swinging motion about two horizontal axes (i.e., the roll $\overrightarrow{\mathbf{b}}_{\mathrm{Bx}}$ and pitch $\overrightarrow{\mathbf{b}}_{\mathrm{By}}$ axes). Although the balance of the sphere is adjusted through trial and error using the balancing mechanisms aligned in the three axes as well as the counterweights, the offset cannot be completely eliminated. Even when the offset was decreased to $1.0 \mathrm{~mm}$, a large gravitational torque of 0.24 $\mathrm{Nm}$ was induced about the horizontal axes when the attitude angle was $1.0^{\circ}$. The frictional torque between the sphere and the saucer was experimentally identified to be approximately $10 \mathrm{mNm}$, which is smaller than the gravitational torque. The CMG system is required to produce control torques about three axes against these disturbance torques.

\subsection{CMGs mounted in the dynamics simulator}

Each CMG consists of a rotor motor to rotate a large flywheel, a gimbal motor, an optical rotary encoder contained in the rotor motor, a resolver contained in the gimbal motor, casing, etc. The maximum gyroscopic output torque produced by each $\mathrm{CMG}$ was $\mathrm{T}_{\mathrm{i}}^{\max }=\mathrm{h}_{\mathrm{Ri}}^{\max } \cdot \dot{\delta}_{\mathrm{i}}^{\max }= \pm 54 \mathrm{Nm}(\mathrm{i}=1-4)$ because the maximum angular momentum and gimbal rate were $\mathrm{h}_{\mathrm{Ri}}^{\max }=54 \mathrm{Nms}$ and $\dot{\delta}_{\mathrm{i}}^{\max }= \pm 1.0 \mathrm{rad} / \mathrm{s}$, respectively. ${ }^{4)}$ The gimbal rotates within the range of $-\pi \leq \delta_{i} \leq \pi \mathrm{rad}$.

The on-board computer includes software with command generation functions from the implemented attitude control and steering laws and data acquisition. The operator can send a command from the host computer, which is connected to the on-board computer through a wireless network connection. The control law generates the torque command to control the sphere's attitude. Subsequently, the gimbal rate command for driving the gimbals is calculated using the steering law, and the gimbals are steered to produce gyroscopic torques. Consequently, the CMG system exerts three-axis control torques on the sphere. The resulting attitude angle and rate of the sphere were measured using a fiber-optic gyro (FOG) sensor, which was also mounted in the lower hemisphere (Fig. 2).

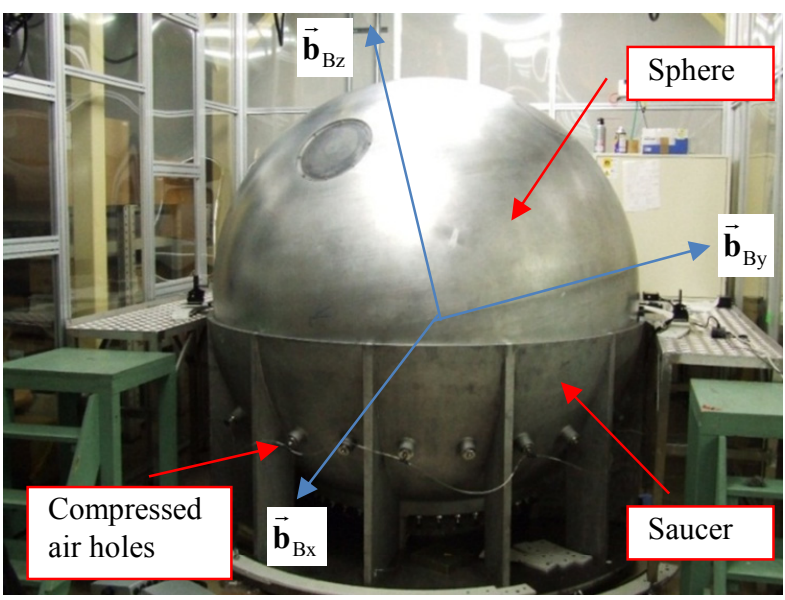

Fig. 1. Dynamics simulator

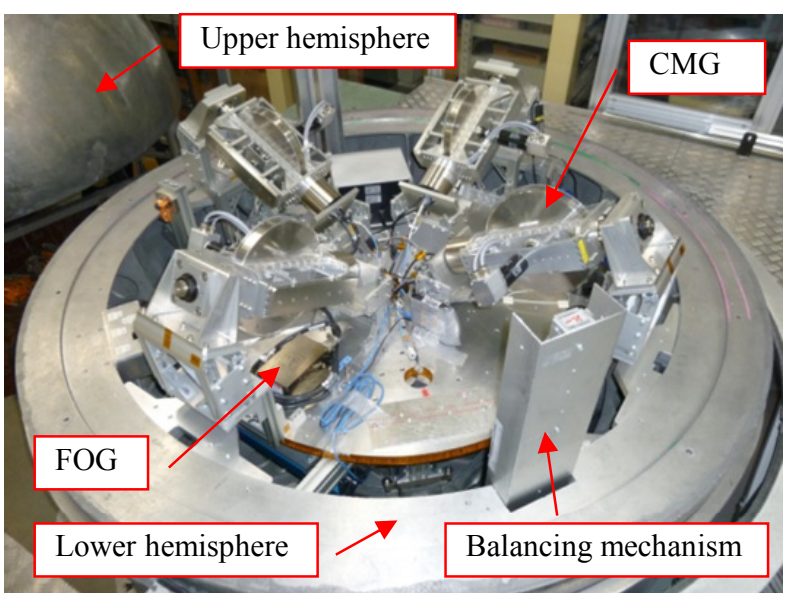

Fig. 2. Four CMGs mounted in the lower hemisphere. 


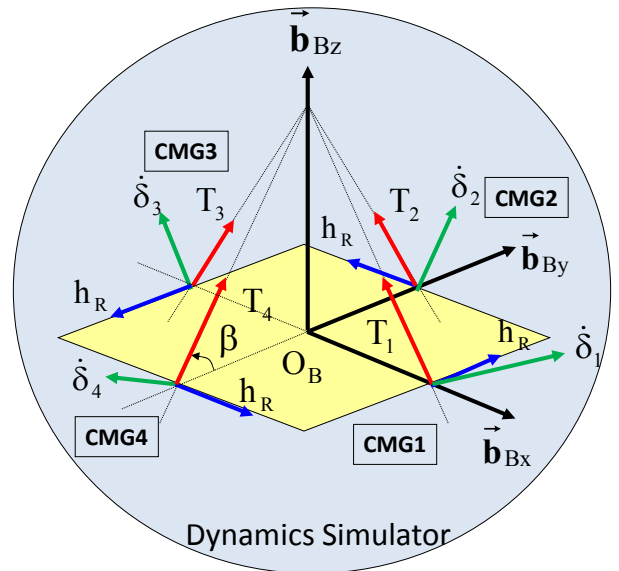

Fig. 3. CMG configuration.

\section{Overview of Dynamics and Control}

\subsection{Equation of motion}

The equation of motion for the sphere, which is equipped with a cluster of four CMGs, is given in the body-fixed reference frame, $\left\{\overrightarrow{\mathbf{b}}_{\mathrm{B}}\right\}^{\mathrm{T}}=\left\{\begin{array}{lll}\overrightarrow{\mathbf{b}}_{\mathrm{Bx}} & \overrightarrow{\mathbf{b}}_{\mathrm{By}} & \overrightarrow{\mathbf{b}}_{\mathrm{Bz}}\end{array}\right\}$ (Fig. 3).

$$
\mathbf{I} \dot{\boldsymbol{\omega}}+\widetilde{\boldsymbol{\omega}} \mathbf{I} \boldsymbol{\omega}+(\dot{\mathbf{H}}+\widetilde{\boldsymbol{\omega}} \mathbf{H})=\mathbf{T}_{\mathrm{DIS}}
$$

The CMG system produces an internal output torque $\dot{\mathbf{H}}+\widetilde{\boldsymbol{\omega}} \mathbf{H} \equiv-\mathbf{T}_{\mathrm{CMG}} \in \mathrm{R}^{3 \times 1}$ to control the sphere's attitude. $\mathbf{H} \in \mathrm{R}^{3 \times 1}$ is the total angular momentum produced by the CMG system, and $\dot{\mathbf{H}} \in \mathrm{R}^{3 \times 1}$ is the time derivative of $\mathbf{H}$. $\mathbf{T}_{\text {DIS }}$ is an external disturbance, such as gravitational and frictional torques. $\dot{\mathbf{H}}$ is given by a Jacobian matrix $\mathbf{A} \in \mathrm{R}^{3 \times 4}$.

$$
\begin{gathered}
\dot{\mathbf{H}}=\mathrm{h}_{\mathrm{R}} \mathrm{A}\left(\beta, \delta_{\mathrm{i}}\right) \cdot \dot{\boldsymbol{\delta}} \\
=\mathrm{h}_{\mathrm{R}}\left[\begin{array}{cccc}
-\mathrm{c} \beta \mathrm{c} \delta_{1} & \mathrm{~s} \delta_{2} & \mathrm{c} \beta \mathrm{c} \delta_{3} & -\mathrm{s} \delta_{4} \\
-\mathrm{s} \delta_{1} & -\mathrm{c} \beta \mathrm{c} \delta_{2} & \mathrm{~s} \delta_{3} & \mathrm{c} \beta \mathrm{c} \delta_{4} \\
\mathrm{~s} \beta \mathrm{c} \delta_{1} & \mathrm{~s} \beta \mathrm{c} \delta_{2} & \mathrm{~s} \beta \mathrm{c} \delta_{3} & \mathrm{~s} \beta \mathrm{c} \delta_{4}
\end{array}\right]\left[\begin{array}{l}
\dot{\delta}_{1} \\
\dot{\delta}_{2} \\
\dot{\delta}_{3} \\
\dot{\delta}_{4}
\end{array}\right]
\end{gathered}
$$

where $\mathrm{c} \beta=\cos \beta, \mathrm{s} \beta=\sin \beta, \mathrm{c} \delta_{\mathrm{i}}=\cos \delta_{\mathrm{i}}$, and $\mathrm{s} \delta_{\mathrm{i}}=\sin \delta_{\mathrm{i}}$ ( $i=1$ - 4). The torque command $\mathbf{u} \in \mathrm{R}^{3 \times 1}$ required for attitude control is expressed as follows:

$$
\mathbf{u}=\dot{\mathbf{H}}=-\left(\mathbf{T}_{\mathrm{CNT}}^{\mathrm{ff}}+\mathbf{T}_{\mathrm{CNT}}^{\mathrm{fb}}\right)-\widetilde{\boldsymbol{\omega}} \mathbf{H}
$$

where $\mathbf{T}_{\mathrm{CNT}}^{\mathrm{ff}} \in \mathrm{R}^{3 \times 1}$ is the feedforward torque command generated as a prescribed profile and $\mathbf{T}_{\mathrm{CNT}}^{\mathrm{fb}} \in \mathrm{R}^{3 \times 1}$ is the feedback torque command, which will be discussed in the subsequent sections.

\subsection{Singularity-robust based steering law}

Some existing CMG steering methods in multitarget attitude maneuvers involve initial gimbal reorientation before slewing and settling. It is assumed that the gimbal angles are positioned at desirable initial gimbal angles using solo null motion, ${ }^{10)}$ namely, a state where the gimbals produce no net torque on the spacecraft. In fact, while the time required to complete gimbal reorientation is shorter than that to complete slewing for each maneuver, the gimbal reorientation time would be impossible to ignore if the maneuvers are repeated and would hinder multitarget observations. Kanzawa et al. ${ }^{5}$ proposed a gimbal steering strategy to eliminate the gimbal reorientation time, and they presented it as a steering law, where the null motion to control the gimbals to the final target gimbal angles is superimposed on the torqued motion to provide the required attitude control torque as follows:

$$
\dot{\boldsymbol{\delta}}_{\mathrm{V}}=\dot{\boldsymbol{\delta}}_{\mathrm{TV}}+\dot{\boldsymbol{\delta}}_{\mathrm{NV}}=\frac{1}{\mathrm{~h}_{\mathrm{R}}} \mathbf{A}^{\#} \cdot \mathbf{u}+\mathrm{K}_{\mathrm{N}} \mathbf{S}_{\mathrm{V}} \boldsymbol{\delta}_{\mathrm{err}}
$$

The first term $\dot{\boldsymbol{\delta}}_{\mathrm{TV}} \in \mathrm{R}^{4 \times 1}$ is the torqued motion based on the singularity-robust steering law-such as the singularity-robust (SR) inverse, ${ }^{11)}$ singular direction avoidance (SDA), ${ }^{12)}$ and generalized singularity-robust (GSR) inverse. ${ }^{13)}$ The second term $\dot{\boldsymbol{\delta}}_{\mathrm{NV}} \in \mathrm{R}^{4 \times 1}$ is the null motion to control the gimbal angles to the pre-determined constant final angles at the end of each maneuver. $\boldsymbol{\delta}_{\text {err }}=\boldsymbol{\delta}_{\mathrm{F}}-\boldsymbol{\delta} \in \mathrm{R}^{4 \times 1}$ denotes the error between the final target angles $\boldsymbol{\delta}_{\mathrm{F}}$ and the current angles $\boldsymbol{\delta}$. The scalar $K_{N}(>0)$ is a null motion gain that represents the amount of null motion. The singularity-robust based steering law does not explicitly avoid singularities but rather approaches and traverses them. Both torqued and null motions can be finite even when the CMG system encounters a singularity because the weighting matrix $\mathbf{V}$ guarantees the non-singularity of the matrices $\mathbf{A}^{\#}=\mathbf{A}^{\mathrm{T}}\left(\mathbf{A} \mathbf{A}^{\mathrm{T}}+\mathbf{V}\right)^{-1} \in \mathrm{R}^{4 \times 3}$ and $\mathbf{S}_{\mathrm{V}}=\mathbf{U}_{44}-\mathbf{A}^{\mathrm{T}}\left(\mathbf{A} \mathbf{A}^{\mathrm{T}}+\mathbf{V}\right)^{-1} \mathbf{A} \in \mathrm{R}^{4 \times 4}$. Kanzawa et al. ${ }^{5)}$ showed that the proposed steering law can reduce singularity encounters without initial gimbal reorientation and provides better performance than GSR using solo torqued motion. However, the singularity-robust based steering law has one drawback, i.e., it induces torque error due to an increase in the weighting matrix $\mathbf{V}$, meaning that the actual output torque produced by the CMG system is not identical to the required torque command $\mathbf{u}$ in the vicinity of a singularity. The following section aims to mitigate this torque error.

\section{Control and Steering Laws Considering Torque Error}

\subsection{Torque error along the singular direction}

Torque error should be minimized because it causes undesirable attitude errors with respect to the prescribed attitude profiles. If the control law generates a large feedback torque command $\mathbf{T}_{\mathrm{CNT}}^{\mathrm{fb}}$ [Eq. (3)] to compensate for attitude errors, the steering law will inevitably generate a command with an undesirable excessive gimbal rate $\dot{\boldsymbol{\delta}}_{\mathrm{TV}}$ [Eq. (4)] because of the large required torque command $\mathbf{u}$. An excessive gimbal rate, involving abrupt changes, will result in gimbal motor load and will unnecessarily consume the gimbal motor's power. Therefore, it is reasonable to provide the steering law with an appropriate feedback torque command to prevent the gimbal rate command from becoming excessive. In contrast, the null motion $\dot{\boldsymbol{\delta}}_{\mathrm{NV}}$ also induces torque error due to the weighting matrix $\mathbf{V}$ because null motion in the vicinity of a singularity is not necessarily in the null space $\left(\mathbf{A} \dot{\boldsymbol{\delta}}_{\mathrm{NV}} \neq \mathbf{O}_{31}\right.$ ). For the reason mentioned above, the second term of Eq. (4) should be modified to produce less torque error.

To reduce the torque error induced by torqued and null 
motion, the feedback torque command $\mathbf{T}_{\mathrm{CNT}}^{\mathrm{fb}}$ and the null motion gain $\mathrm{K}_{\mathrm{N}}$ were modified on the basis of the following analysis. Mathematically, the SVD of the Jacobian matrix is useful for determining the direction and magnitude of the torque error. ${ }^{6}$ The SVD of the Jacobian matrix $\mathbf{A} \in \mathrm{R}^{3 \times 4}$ is generally defined as follows:

$$
\mathbf{A}=\mathbf{U}_{\mathrm{s}} \mathbf{\Sigma} \mathbf{V}_{\mathrm{s}}^{\mathrm{T}}=\sum_{\mathrm{i}=1}^{3} \sigma_{\mathrm{i}} \mathbf{u}_{\mathrm{si}} \mathbf{i}_{\mathrm{si}}^{\mathrm{T}}
$$

where $\sigma_{1} \geq \sigma_{2} \geq \sigma_{3} \geq 0$ are singular values, $\mathbf{u}_{\mathrm{si}} \in \mathrm{R}^{3 \times 1}$ (i= 1-3) is a left singular vector, and $\mathbf{v}_{\mathrm{si}} \in \mathrm{R}^{4 \times 1}(\mathrm{i}=1-4)$ is a right singular vector. $\mathbf{U}_{\mathrm{s}}=\left[\begin{array}{lll}\mathbf{u}_{\mathrm{s} 1} & \mathbf{u}_{\mathrm{s} 2} & \mathbf{u}_{\mathrm{s} 3}\end{array}\right] \in \mathrm{R}^{3 \times 3}$ and $\mathbf{V}_{\mathrm{s}}=\left[\begin{array}{llll}\mathbf{v}_{\mathrm{s} 1} & \mathbf{v}_{\mathrm{s} 2} & \mathbf{v}_{\mathrm{s} 3} & \mathbf{v}_{\mathrm{s} 4}\end{array}\right] \in \mathrm{R}^{4 \times 4}$ are orthogonal matrices. In other words, $\mathbf{U}_{\mathrm{s}} \mathbf{U}_{\mathrm{s}}^{\mathrm{T}}=\mathbf{U}_{\mathrm{s}}^{\mathrm{T}} \mathbf{U}_{\mathrm{s}}=\mathbf{U}_{33}$ and $\mathbf{V}_{\mathrm{s}} \mathbf{V}_{\mathrm{s}}^{\mathrm{T}}=\mathbf{V}_{\mathrm{s}}^{\mathrm{T}} \mathbf{V}_{\mathrm{s}}=\mathbf{U}_{44}$. An expression of the diagonal weighting matrix $\mathbf{V}$, using the left singular vector $\mathbf{u}_{\mathrm{si}}$, is introduced here. ${ }^{6}$

$$
\mathbf{V}=\mathbf{U}_{\mathrm{s}} \mathbf{D} \mathbf{U}_{\mathrm{s}}^{\mathrm{T}}=\sum_{\mathrm{i}=1}^{3} \mathrm{~d}_{\mathrm{i}}^{2} \mathbf{u}_{\mathrm{si}} \mathbf{u}_{\mathrm{si}}^{\mathrm{T}}
$$

where $d_{i}(i=1-3)$ is the singularity robustness parameter. Using Eqs. (5) and (6), the matrices $\mathbf{A}^{\#}$ and $\mathbf{S}_{\mathrm{V}}$ are, respectively, transformed to

$$
\begin{gathered}
\mathbf{A}^{\#}=\sum_{\mathrm{i}=1}^{3} \frac{\sigma_{\mathrm{i}}}{\sigma_{\mathrm{i}}^{2}+\mathrm{d}_{\mathrm{i}}^{2}} \mathbf{v}_{\mathrm{si}} \mathbf{u}_{\mathrm{si}}^{\mathrm{T}} \\
\mathbf{S}_{\mathrm{V}}=\sum_{\mathrm{i}=1}^{3} \frac{\mathrm{d}_{\mathrm{i}}^{2}}{\sigma_{\mathrm{i}}^{2}+\mathrm{d}_{\mathrm{i}}^{2}} \mathbf{v}_{\mathrm{si}} \mathbf{v}_{\mathrm{si}}^{\mathrm{T}}+\mathbf{v}_{\mathrm{s} 4} \mathbf{v}_{\mathrm{s} 4}^{\mathrm{T}}
\end{gathered}
$$

Substituting Eqs. (7) and (8) into Eq. (4), the steering law is represented by a linear combination of right singular vectors $\mathbf{v}_{\mathrm{si}}$.

$$
\begin{gathered}
\dot{\boldsymbol{\delta}}_{\mathrm{V}}=\dot{\boldsymbol{\delta}}_{\mathrm{TV}}+\dot{\boldsymbol{\delta}}_{\mathrm{NV}} \\
=\frac{1}{\mathrm{~h}_{\mathrm{R}}} \sum_{\mathrm{i}=1}^{3} \frac{\sigma_{\mathrm{i}} \mathbf{u}_{\mathrm{si}}^{\mathrm{T}} \mathbf{u}_{\mathrm{i}}^{2}+\mathrm{d}_{\mathrm{i}}^{2}}{\mathbf{v}_{\mathrm{si}}}+\mathrm{K}_{\mathrm{N}}\left(\sum_{\mathrm{i}=1}^{3} \frac{\mathrm{d}_{\mathrm{i}}^{2} \mathbf{v}_{\mathrm{si}}^{\mathrm{T}} \boldsymbol{\delta}_{\mathrm{err}}}{\sigma_{\mathrm{i}}^{2}+\mathrm{d}_{\mathrm{i}}^{2}} \mathbf{v}_{\mathrm{si}}+\mathbf{v}_{\mathrm{s} 4}^{\mathrm{T}} \boldsymbol{\delta}_{\mathrm{err}} \mathbf{v}_{\mathrm{s} 4}\right)
\end{gathered}
$$

As the gimbal angles approach a singular configuration, the smallest singular value becomes zero $\left(\sigma_{3} \rightarrow 0\right)$. The parameter $\mathrm{d}_{\mathrm{i}}$ becomes large to prevent torqued motion $\dot{\boldsymbol{\delta}}_{\mathrm{TV}}$ and null motion $\dot{\boldsymbol{\delta}}_{\mathrm{NV}}$ from becoming infinite, thereby causing torque error. If the parameters are chosen as $d_{i}=d \neq 0 \quad(i=1-3)$, the first term in Eq. (9) corresponds to $S R,{ }^{11)}$ if the parameters are $d_{1}=d_{2}=0$ and $d_{3} \neq 0$, it corresponds to SDA. ${ }^{12}$

Here, the torque command $\mathbf{u}$ is defined as a linear combination of left singular vectors $\mathbf{u}_{\mathrm{si}}{ }^{6,15)}$

$$
\mathbf{u}=\sum_{\mathrm{i}=1}^{3} \zeta_{\mathrm{i}} \mathbf{u}_{\mathrm{si}}
$$

where $\zeta_{i}(i=1-3)$ is the magnitude of the torque command $\mathbf{u}$ along each direction $\mathbf{u}_{\mathrm{si}}$. Multiplying $\mathrm{h}_{\mathrm{R}} \mathbf{A}$ from the left-hand side of Eq. (9), the actual output torque $\mathbf{u}_{\mathrm{d}}$ produced by the CMG system is obtained. The torque error $\mathbf{u}_{\text {err }}$ is defined as the difference between the torque command $\mathbf{u}$ and the actual output torque $\mathbf{u}_{\mathrm{d}}$, which is expressed as follows:

$$
\begin{gathered}
\mathbf{u}_{\text {err }}=\mathbf{u}-\mathbf{u}_{\mathrm{d}}=\left(\mathbf{u}-\mathrm{h}_{\mathrm{R}} \mathbf{A} \dot{\boldsymbol{\delta}}_{\mathrm{TV}}\right)-\mathrm{h}_{\mathrm{R}} \mathbf{A} \dot{\boldsymbol{\delta}}_{\mathrm{NV}} \\
=\sum_{\mathrm{i}=1}^{3} \frac{\mathrm{d}_{\mathrm{i}}^{2} \zeta_{\mathrm{i}}}{\sigma_{\mathrm{i}}^{2}+\mathrm{d}_{\mathrm{i}}^{2}} \mathbf{u}_{\mathrm{si}}-\mathrm{h}_{\mathrm{R}} \mathrm{K}_{\mathrm{N}} \sum_{\mathrm{i}=1}^{3} \frac{\sigma_{\mathrm{i}} \mathrm{d}_{\mathrm{i}}^{2} \mathbf{v}_{\mathrm{si}}^{\mathrm{T}} \boldsymbol{\boldsymbol { o }}_{\mathrm{err}}}{\sigma_{\mathrm{i}}^{2}+\mathrm{d}_{\mathrm{i}}^{2}} \mathbf{u}_{\mathrm{si}}
\end{gathered}
$$

To reduce the torque error, it is reasonable to analyze the torque error based on SDA, ${ }^{12)}$ which guarantees a smaller torque error than that produced with SR. Thus, the steering law of Eq. (9) uses only the third parameter, i.e., $d_{3}$, which varies with its distance from the singularity.

$$
\mathrm{d}_{1}=\mathrm{d}_{2}=0 \text { and } \mathrm{d}_{3}=\sqrt{\lambda_{\mathrm{v} 0} \exp \left(-\mu_{\mathrm{v}} \mathrm{m}^{2}\right)}
$$

where $\lambda_{\mathrm{v} 0}$ and $\mu_{\mathrm{v}}$ are positive constant scalar values and $\mathrm{m}$ is the so-called CMG gain, a measure of how close the gimbal configuration is to the singularity.

$$
\mathrm{m}=\sqrt{\operatorname{det}\left(\mathbf{A} \mathbf{A}^{\mathrm{T}}\right)}=\sqrt{\operatorname{det}\left(\boldsymbol{\Sigma} \boldsymbol{\Sigma}^{\mathrm{T}}\right)}=\sqrt{\prod_{\mathrm{i}=1}^{3} \sigma_{\mathrm{i}}^{2}}
$$

Consequently, the resulting torque error induced by the combined torqued and null motion steering law based on SDA can be expressed in terms of only a singular direction $\mathbf{u}_{\mathrm{s} 3}$, i.e.,

$$
\begin{gathered}
\mathbf{u}_{\mathrm{err}}=\left(\xi_{\mathrm{T} 3}+\xi_{\mathrm{N} 3}\right) \mathbf{u}_{\mathrm{s} 3} \\
=\left(\frac{\mathrm{d}_{3}^{2}}{\sigma_{3}^{2}+\mathrm{d}_{3}^{2}} \zeta_{3}-\frac{\sigma_{3} \mathrm{~d}_{3}^{2}}{\sigma_{3}^{2}+\mathrm{d}_{3}^{2}} \mathrm{~h}_{\mathrm{R}} \mathrm{K}_{\mathrm{N}} \mathbf{v}_{\mathrm{s} 3}^{\mathrm{T}} \boldsymbol{\delta}_{\mathrm{err}}\right) \mathbf{u}_{\mathrm{s} 3}
\end{gathered}
$$

We determined that the torque error $\xi_{\text {тз }}$ induced by torqued motion $\dot{\boldsymbol{\delta}}_{\mathrm{TV}}$ appears to be proportional to the magnitude of the torque command $\zeta_{3}$ and that the torque error $\xi_{\mathrm{N} 3}$ induced by null motion $\dot{\boldsymbol{\delta}}_{\mathrm{NV}}$ appears to be proportional to the magnitude of the null motion gain $\mathrm{K}_{\mathrm{N}}$. To reduce the second term in Eq. (14), the gain $\mathrm{K}_{\mathrm{N}}$ is modified as follows:

$$
\mathrm{K}_{\mathrm{N}}^{\mathrm{m}}(\mathrm{m})=\mathrm{K}_{\mathrm{N} 0}\left\{1-\mathrm{k}_{\mathrm{N}} \exp \left(-\mu_{\mathrm{N}} \mathrm{m}^{2}\right)\right\}
$$

where $\mathrm{K}_{\mathrm{N} 0}, \mathrm{k}_{\mathrm{N}} \leq 1$, and $\mu_{\mathrm{N}}$ are positive constant scalar values. As the gimbal angles approach a singular configuration $(\mathrm{m} \rightarrow 0)$, the null motion gain $\mathrm{K}_{\mathrm{N}}^{\mathrm{m}}$ decreases to reduce the torque error.

\subsection{Modified feedback control law}

In the following analysis, a feedback torque command to reduce the first term in Eq. (14) is proposed. Similar to Eq. (10), the feedback torque command given by a simple PD control law is represented by

$$
\mathbf{T}_{\mathrm{CNT}}^{\mathrm{fb}}=\mathbf{K}_{\mathrm{d}}\left(\boldsymbol{\omega}_{\mathrm{REF}}-\boldsymbol{\omega}\right)+\mathbf{K}_{\mathrm{p}}\left(\boldsymbol{\Theta}_{\mathrm{REF}}-\boldsymbol{\Theta}\right)=\sum_{\mathrm{i}=1}^{3} \zeta_{\mathrm{i}}^{\mathrm{fb}} \mathbf{u}_{\mathrm{si}}
$$

where $\boldsymbol{\omega}_{\mathrm{REF}} \in \mathrm{R}^{3 \times 1}$ and $\boldsymbol{\Theta}_{\mathrm{REF}} \in \mathrm{R}^{3 \times 1}$ are the attitude rate and angle profiles given by the feedback control torque [ $\mathbf{T}_{\mathrm{CNT}}^{\mathrm{ff}}$ in Eq. (3)], respectively. $\mathbf{K}_{\mathrm{d}}=\operatorname{diag}\left[\mathrm{k}_{\mathrm{dx}} \quad \mathrm{k}_{\mathrm{dy}} \mathrm{k}_{\mathrm{dz}}\right]$ and $\mathbf{K}_{\mathrm{p}}=\operatorname{diag}\left[\begin{array}{lll}\mathrm{k}_{\mathrm{px}} & \mathrm{k}_{\mathrm{py}} & \mathrm{k}_{\mathrm{pz}}\end{array}\right]$ are the constant derivative and proportional gain matrices, respectively. Here the following function in terms of the CMG gain $\mathrm{m}$ is defined for the singular direction $\mathbf{u}_{\mathrm{s} 3}$ :

$$
\zeta_{\mathrm{m} 3}^{\mathrm{fb}}(\mathrm{m}) \mathbf{u}_{\mathrm{s} 3} \equiv \zeta_{3}^{\mathrm{fb}} \mathrm{k}_{\mathrm{T} 3} \exp \left(-\mu_{\mathrm{T} 3} \mathrm{~m}^{2}\right) \mathbf{u}_{\mathrm{s} 3}
$$


where $\mathrm{k}_{\mathrm{T} 3} \leq 1$ and $\mu_{\mathrm{T} 3}$ are positive constant scalar values. Subtracting Eq. (17) from Eq. (16), the modified feedback torque command, depending on the $\mathrm{CMG}$ gain $\mathrm{m}$, is represented along with Eq. (15):

$$
\begin{gathered}
\mathbf{T}_{\mathrm{CNT}}^{\mathrm{fbm}}(\mathrm{m})=\mathbf{K}_{\mathrm{d}}\left(\boldsymbol{\omega}_{\mathrm{REF}}-\boldsymbol{\omega}\right)+\mathbf{K}_{\mathrm{p}}\left(\boldsymbol{\Theta}_{\mathrm{REF}}-\boldsymbol{\Theta}\right)-\zeta_{\mathrm{m} 3}^{\mathrm{fb}}(\mathrm{m}) \mathbf{u}_{\mathrm{s} 3} \\
=\zeta_{1}^{\mathrm{fb}} \mathbf{u}_{\mathrm{s} 1}+\zeta_{2}^{\mathrm{fb}} \mathbf{u}_{\mathrm{s} 2}+\zeta_{3}^{\mathrm{fb}}\left\{1-\mathrm{k}_{\mathrm{T} 3} \exp \left(-\mu_{\mathrm{T} 3} \mathrm{~m}^{2}\right)\right\} \mathbf{u}_{\mathrm{s} 3}
\end{gathered}
$$

Figure 4 illustrates the feedback torque command regulated by the $\mathrm{CMG}$ gain. When the gimbal configuration is close to a singularity, the magnitude of the feedback torque command along the singular direction $\mathbf{u}_{\mathrm{s} 3}$ decreases, while the torque command along the orthogonal axis $\zeta_{1}^{\mathrm{fb}} \mathbf{u}_{\mathrm{s} 1}+\zeta_{2}^{\mathrm{fb}} \mathbf{u}_{\mathrm{s} 2}$ maintains its magnitude. In other words, the proposed control law works as a torque limiter in the vicinity of a singularity. The numerical simulations performed by Kanzawa et al. ${ }^{14)}$ demonstrate that the control law [Eq. (3)] with the feedback torque command [Eq. (18)], and the steering law [Eq. (9)] with the null motion gain [Eq. (15)] are able to reduce the torque error and gimbal rate.

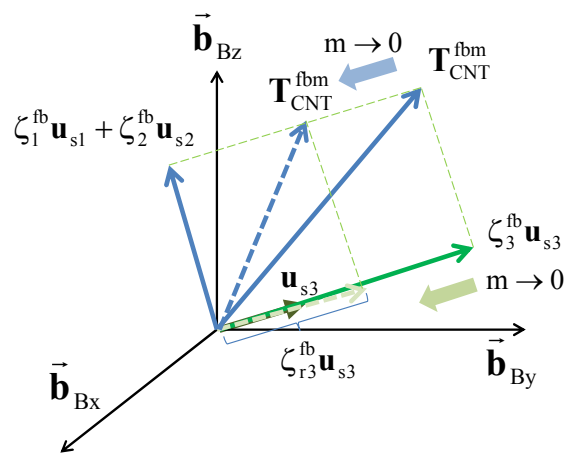

Fig. 4. Feedback torque command regulated by the CMG gain.

\section{Preliminary Simulation Before Maneuver Tests}

As the first step of our agile maneuver tests using the dynamics simulator, we performed demonstration tests about the yaw axis $\overrightarrow{\mathbf{b}}_{\mathrm{Bz}}$, which are easier to conduct than those about the horizontal axes because the yaw axis suffers from lower gravitational torque. Before the demonstration tests, preliminary simulations were performed to create maneuver profiles and predict the movement range of the gimbals. The profiles $\mathbf{T}_{\mathrm{CNT}}^{\mathrm{ff}}, \boldsymbol{\omega}_{\mathrm{REF}}$, and $\boldsymbol{\Theta}_{\mathrm{REF}}$ about the yaw axis were determined to ensure that the maneuver test satisfied the conditions of a large angle $\left(60^{\circ}\right)$ and a high rate $(3 \mathrm{deg} / \mathrm{s})$. Here, actual hardware constraints for the gimbal angle $-\pi \leq \delta_{\mathrm{i}} \leq \pi \mathrm{rad}$. and the gimbal rate $-1.0 \leq \dot{\delta}_{\mathrm{i}} \leq 1.0 \mathrm{rad} / \mathrm{s}$ were considered under the condition that the individual rotor angular momentum was set to $\mathrm{h}_{\mathrm{R}}=10 \mathrm{Nms}$. Furthermore, the dynamics model, control law, and steering law presented in the previous sections were simplified using the following assumptions:

a) The simulation excludes disturbance torques such as those caused by gravity and friction $\left[\mathbf{T}_{\mathrm{DIS}}=\mathbf{O}_{31}\right.$ in Eq. (1)].

b) For simplicity, the gimbal angles stayed away from a singular configuration, and null motion was not applied.
Assumption a) means that the feedback control torque to compensate for attitude errors due to disturbance torques was almost zero and that the gimbals did not respond to the feedback torque command. Assumption b) means that the implementation of the pseudo inverse law is sufficient $\left[d_{i}=0\right.$ and $\mathrm{K}_{\mathrm{N}}=0$ in Eq. (9)]; only the feedforward torque command [ $\mathbf{T}_{\mathrm{CNT}}^{\mathrm{ff}}$ in Eq. (3)] was applied as a prescribed profile because of assumption a).

$$
\begin{gathered}
\mathbf{T}_{\mathrm{CNT}}^{\mathrm{ff}}=\mathrm{I}_{\mathrm{z}} \ddot{\psi} \cdot \mathbf{e} \\
=\mathrm{I}_{\mathrm{z}}\left(12 \mathrm{p}_{4} \mathrm{t}^{2}+20 \mathrm{p}_{5} \mathrm{t}^{3}+30 \mathrm{p}_{6} \mathrm{t}^{4}+42 \mathrm{p}_{7} \mathrm{t}^{5}\right) \cdot \mathbf{e}
\end{gathered}
$$

where $I_{z}=615 \mathrm{kgm}^{2}$ is the moment of inertia, $\ddot{\psi}$ is the angular acceleration profile about the yaw axis $\mathbf{e}=\left[\begin{array}{lll}0 & 0 & 1\end{array}\right]^{\mathrm{T}}$, and $\mathrm{p}_{4}, \mathrm{p}_{5}, \mathrm{p}_{6}$, and $\mathrm{p}_{7}$ are the parameters, including the target maneuver angle $\psi_{\mathrm{m}}$ and the maneuver time $\mathrm{t}_{\mathrm{m}} \cdot{ }^{4)}$

Figure 5 illustrates the preliminary simulation results for the maneuver test. As shown in Fig. 5(a), the first maneuver was performed from an initial attitude of $\boldsymbol{\Theta}=\left[\begin{array}{lll}0 & 0 & 0\end{array}\right]^{\mathrm{T}} \mathrm{deg}$ to a target final attitude $\boldsymbol{\Theta}=\left[\begin{array}{lll}0 & 0 & 60\end{array}\right]^{\mathrm{T}}$ deg during $0 \leq \mathrm{t} \leq 45 \mathrm{~s}$. Subsequently, the second maneuver was performed in the opposite direction during $50 \leq \mathrm{t} \leq 95 \mathrm{~s}$. As shown in Fig. 5(b), the maximum attitude rate reached approximately $3 \mathrm{deg} / \mathrm{s}$. Figures 5(c)-(e) show the torque command $\mathbf{T}_{\mathrm{CNT}} \equiv \mathbf{T}_{\mathrm{CNT}}^{\mathrm{ff}}+\mathbf{T}_{\mathrm{CNT}}^{\mathrm{fb}}$, the total output torque $\dot{\mathbf{H}}$, and the total angular momentum $\mathbf{H}$, respectively. From Eq. (3), the relation $\dot{\mathbf{H}}=-\mathbf{T}_{\mathrm{CNT}}$ is satisfied because of the single yaw axis maneuver. Figures 5(f) and (g) show that the gimbals are simultaneously steered by the same gimbal rate command calculated by the pseudo inverse law.

\section{Maneuver Demonstration Tests}

The simplified control and steering laws described in Section 5 were implemented in the dynamics simulator with the same maneuver profiles and rotor angular momentum. The feedback gains for each axis were chosen to be $\mathrm{k}_{\mathrm{d}}=27.63$ $\mathrm{Nms} / \mathrm{rad}$ and $\mathrm{k}_{\mathrm{p}}=29.83 \mathrm{Nm} / \mathrm{rad}$ since the control bandwidth was set to $0.035 \mathrm{~Hz}$. Before the maneuver demonstration test, swing motion due to gravitational torque was stabilized using counterweights in the lower hemisphere. The resulting natural frequency due to gravitational torque was $0.05 \mathrm{~Hz}$, which is beyond the control bandwidth.

Figure 6 illustrates the maneuver test results. Figures 6(a) and (b) show that the sphere's attitude angle and rate were controlled based on the prescribed profiles. Since the actual time histories about the yaw axis, which are shown in Figs. 6(c)-(e), were identical to those predicted by the simulation, we confirmed that the CMG system produced the expected total angular momentum $\mathbf{H}$ and output torque $\dot{\mathbf{H}}$ during the maneuvers. Because this test is based on condition b), torque error due to the singularity is not produced. Thus, Fig. 7 shows that the torque error $\left(\mathbf{u}_{\mathrm{err}}=\dot{\mathbf{H}}-\left(-\mathbf{T}_{\mathrm{CNT}}\right)\right)$ between the torque command [Fig. 6(c)] and the actual output torque [Fig. $6(d)]$ is small. 

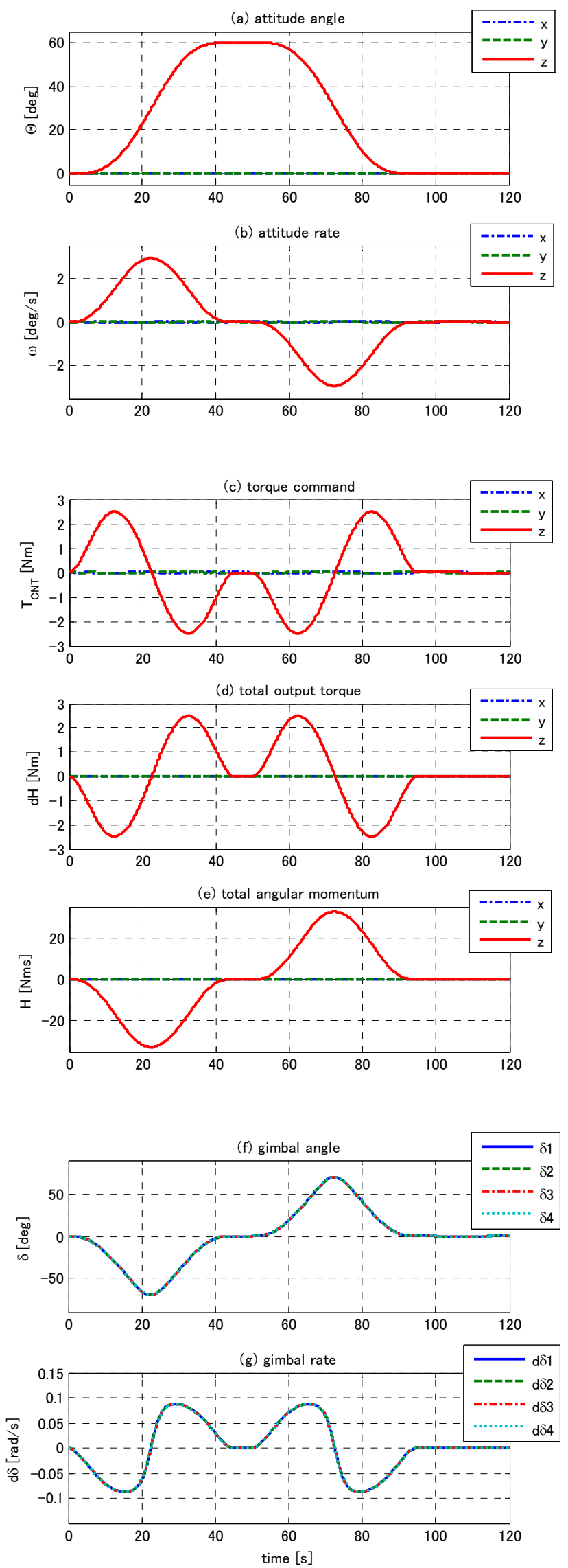

Fig. 5. Preliminary simulation results.
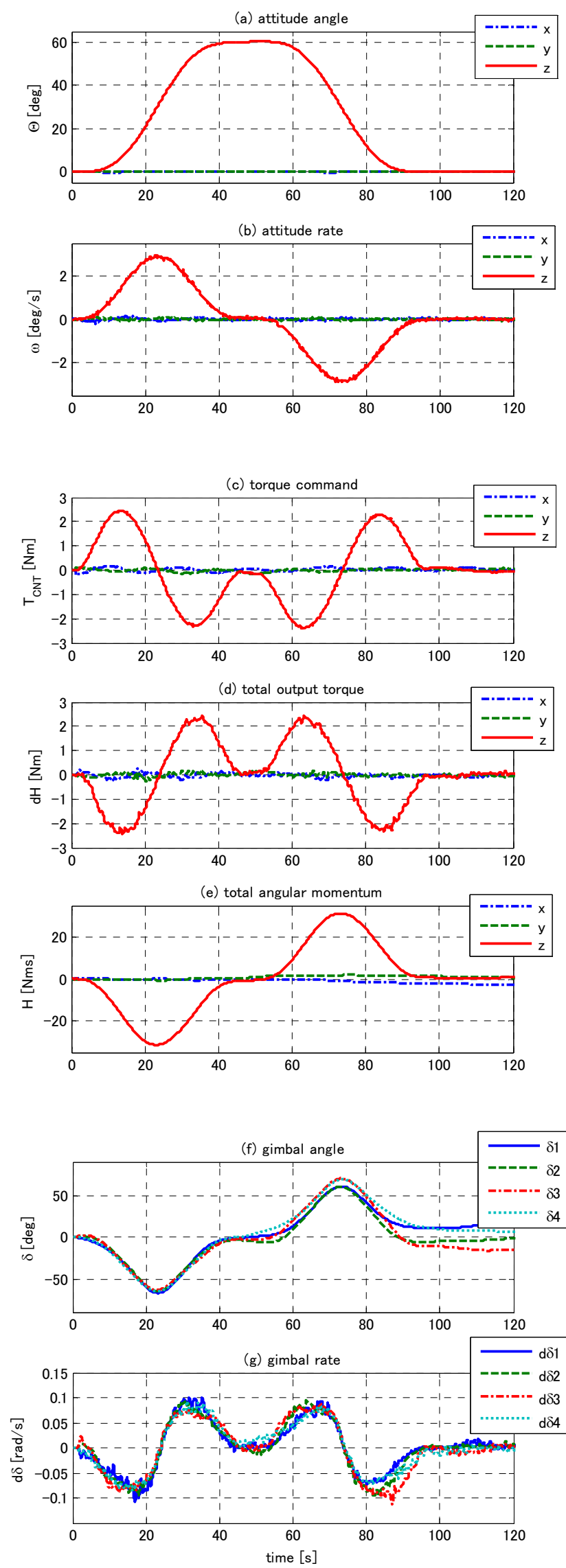

Fig. 6. Maneuver demonstration test results. 


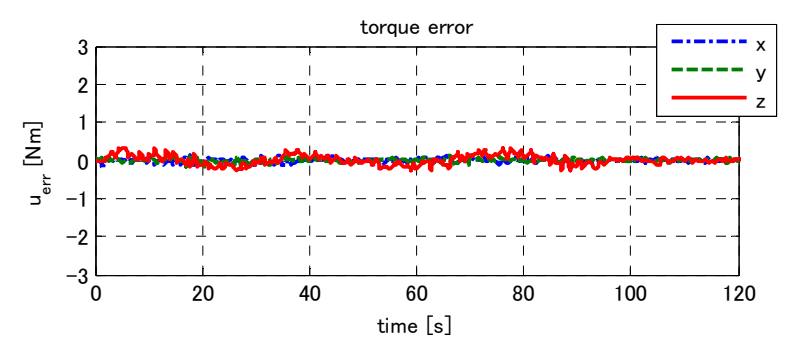

Fig. 7. Torque error in maneuver demonstration test.
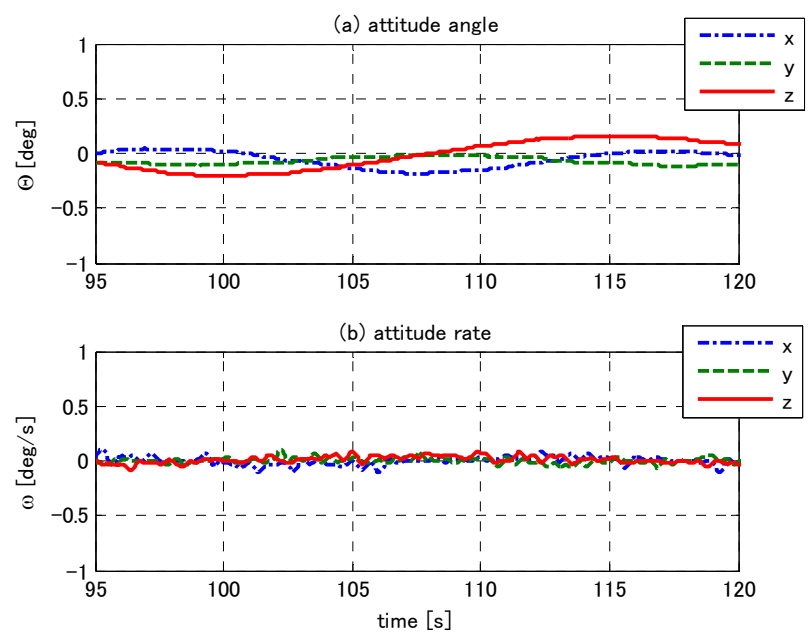

Fig. 8. Attitude angle and rate at the end of the second maneuver in the demonstration test.

Conversely, the actual behavior of the gimbal angles and rates, which are shown in Figs. 6(f) and (g), differ from the numerical prediction; this discrepancy can be attributed to frictional and gravitational torques because the gimbals were steered against these disturbance torques based on the feedback torque command. It can be seen that the total angular momentum shown in Fig. 6(e) does not converge to zero as well as the gimbal angles due to the frictional and gravitational torques.

At the end of the second maneuver, the attitude angles converged to the target maneuver angles $\boldsymbol{\Theta}=\left[\begin{array}{lll}0 & 0 & 0\end{array}\right]^{\mathrm{T}}$ deg within the maximum error $\boldsymbol{\Theta}_{\text {err }}^{\max }=\left[\begin{array}{lll}0.18 & 0.12 & 0.20\end{array}\right]^{\mathrm{T}}$ deg as shown in Fig. 8(a). Thus, the dynamics simulator successfully accomplished agile, large angle, and rest-to-rest multitarget maneuvers as well as precision pointing.

\section{Conclusion}

We provided an overview of a large dynamics simulator that provides an on-ground test environment for free rotational movement around three axes. The issue of torque error between the torque command and the actual output torque was discussed with regards to the singularity-robust based steering law. The torque error induced by the singularity robustness parameter was analyzed using SVD to determine the error's magnitude and direction. Based on the torque error analysis, feedback control and steering laws for reducing the torque error were proposed. Before the maneuver demonstration tests using the dynamics simulator, a preliminary simulation was performed using the simplified dynamics model, control law, and steering law. The maneuver test results showed that the dynamics simulator successfully accomplished agile, large angle, and rest-to-rest maneuvers as well as precision pointing.

\section{References}

1) Kurokawa, H.: Survey of Theory and Steering Laws of Single-Gimbal Control Moment Gyros, Journal of Guidance, Control, and Dynamics, 30 (2007), pp. 1331-1340.

2) Jones, L. L., Zeledon, R. A. and Peck. M. A.: Generalized Framework for Linearly Constrained Control Moment Gyro Steering, Journal of Guidance, Control, and Dynamics, 35 (2012), pp. 1094-1103.

3) Endo, T., Kawai, H., Kanzawa, T. and Izawa, K.: CMG Test Bed Development, Journal of Space Technology and Science, 25 (2009), pp.69-81.

4) Kanzawa, T., Endo, T., Kawai, H., Fujiwara, K., Kakehashi, Y, Fukuyama, T. and Sasaki, N.: Development of CMG for Three-Axis Free Air Floating Dynamics Simulator, Trans. JSASS Aerospace Tech. Japan, 8 (2010), pp.Pd_69-Pd_74.

5) Kanzawa, T., Haruki, M. and Yamanaka, K.: Steering Law of Control Moment Gyros Using both Torque-Producing Motion and Null Motion for Agile Multitarget Attitude Maneuvers, Proceedings of the 23th Workshop on JAXA Astrodynamics and Flight Mechanics, 2013, pp. 236-244.

6) Jin, J., Zhang, J. and Liu, Z.: Output-Torque Error Analysis and Steering Law Design of SGCMGs Based on SVD Theory, AIAA Guidance, Navigation, and Control Conference, 2009, Chicago, illinois, AIAA 2009-5804.

7) Schwartz, J. L., Peck, M. A. and Hall, C. D.: Historical Review of Air-Bearing Spacecraft Simulators, Journal of Guidance, Control, and Dynamics, 26 (2003), pp. 513-522.

8) Jung, D. and Tsiotras, P.: An Experimental Comparison of CMG Steering Control Laws, AIAA/AAS Astrodynamics Specialist Conference and Exhibit, 2004, AIAA 2004-5294.

9) Underhill, B. and Hamilton, B.: Momentum Control System and Line-of-Sight Testbed, 29th Annual AAS Guidance and Control Conference, 2006, AAS 06-053.

10) Vadali, S. R., Oh, H. S. and Walker S. R.: Preferred Gimbal Angles for Single Gimbal Control Moment Gyros, Journal of Guidance, Control, and Dynamics, 13 (1990), pp. 1090-1095.

11) Bedrossian, N. S., Paradiso, J., Bergmann, E. V. and Rowell, D.: Steering Law Design for Redundant Single-Gimbal Control Moment Gyroscopes, Journal of Guidance, Control, and Dynamics, 13 (1990), pp. 1083-1089.

12) Ford, K. A. and Hall, C. D.: Singular Direction Avoidance Steering for Control-Moment Gyros, Journal of Guidance, Control, and Dynamics, 23 (2000), pp. 648-656.

13) Wie, B., Bailey, D. and Heiberg, C.: Singularity Robust Steering Logic for Redundant Single-Gimbal Control Moment Gyros, Journal of Guidance, Control, and Dynamics, 24 (2001), pp. 865-872.

14) Kanzawa, T., Haruki, M. and Yamanaka, K.: Control and Steering Laws Considering Torque Error in Singularity of Control Moment Gyro, The 2nd Multi-Symposium on Control Systems, 2015, 662-1. (in Japanese)

15) Zhang, J., Ma, K. and Meng, G.: Large Angle Maneuvers of Spacecraft Based on Singularity Avoidance of SGCMGs, AIAA Guidance, Navigation, and Control Conference, 2013, AIAA 2013-4559. 